\title{
BMJ
}

\section{Disparities in breast cancer mortality trends between 30 European countries: retrospective trend analysis of WHO mortality database}

\author{
Philippe Autier, group head and research director, 1,2 Mathieu Boniol, biostatistician and senior researcher, 1,2 \\ Carlo LaVecchia, professor, ${ }^{3}$ Lars Vatten, professor, ${ }^{4}$ Anna Gavin, director of Northern Ireland Cancer \\ Registry, ${ }^{5}$ Clarisse Héry, researcher, ${ }^{1}$ Mary Heanue, senior researcher ${ }^{1}$
}

${ }^{1}$ International Agency for Research on Cancer, Lyon, France

${ }^{2}$ International Prevention Research Institute (iPRI), Lyon, France

${ }^{3}$ Mario Negri Institute and School of Medicine of the University of Milan, Milan, Italy

${ }^{4}$ Department of Public Health, Norwegian University of Science and Technology, Trondheim,

Norway

${ }^{5}$ Northern Ireland Cancer Registry, Queen's University, Belfast, Northern Ireland

Correspondence to: P Autier, International Prevention Research Institute (iPRI), 95 Cours Lafayette, 69006 Lyon, France philippe.autier@i-pri.org

Cite this as: $B M J$ 2010;341:c3620 doi:10.1136/bmj.c3620

\section{ABSTRACT}

Objective To examine changes in temporal trends in breast cancer mortality in women living in 30 European countries. Design Retrospective trend analysis.

Data source WHO mortality database on causes of deaths Subjects reviewed Female deaths from breast cancer from 1989 to 2006

Main outcome measures Changes in breast cancer mortality for all women and by age group $(<50,50-69$, and $\geq 70$ years) calculated from linear regressions of log transformed, age adjusted death rates. Joinpoint analysis was used to identify the year when trends in all age mortality began to change.

Results From 1989 to 2006, there was a median reduction in breast cancer mortality of $19 \%$, ranging from a $45 \%$ reduction in Iceland to a $17 \%$ increase in Romania. Breast cancer mortality decreased by $\geq 20 \%$ in 15 countries, and the reduction tended to be greater in countries with higher mortality in 1987-9. England and Wales, Northern Ireland, and Scotland had the second, third, and fourth largest decreases of 35\%, 29\%, and 30\%, respectively. In France, Finland, and Sweden, mortality decreased by $11 \%, 12 \%$, and $16 \%$, respectively. In central European countries mortality did not decline or even increased during the period. Downward mortality trends usually started between 1988 and 1996, and the persistent reduction from 1999 to 2006 indicates that these trends may continue. The median changes in the age groups were $-37 \%$ (range $-76 \%$ to $-14 \%$ ) in women aged $<50,-21 \%$ $(-40 \%$ to $14 \%)$ in $50-69$ year olds, and $-2 \%(-42 \%$ to $80 \%)$ in $\geq 70$ year olds.

Conclusions Changes in breast cancer mortality after 1988 varied widely between European countries, and the UK is among the countries with the largest reductions. Women aged $<50$ years showed the greatest reductions in mortality, also in countries where screening at that age is uncommon. The increasing mortality in some central European countries reflects avoidable mortality.

\section{INTRODUCTION}

Since the 1990s, profound changes have taken place in the clinical presentation and management of breast cancer due to mammography screening, implementation of effective hormone treatments and chemotherapy, and progress in radiotherapy and surgery. These innovations have probably contributed to the observed improvements in breast cancer survival in Europe, ${ }^{12}$ but the contribution of each factor is unknown. The implementation of mammography screening, the availability of new treatments, and healthcare expenditures vary greatly across Europe..$^{3-7}$ One consequence could be that mortality decreases between countries will also vary, and the highest decreases are likely to be observed in countries characterised by early implementation and high attendance to breast screening combined with rapid uptake of anticancer drugs.

In this study we analysed breast cancer mortality in 30 European countries from 1980 to 2006 and examined temporal trends in rates for all women, and for women aged $<50,50-69$, and $\geq 70$ years to help decipher the effect of the contributing factors.

\section{METHODS}

Breast cancer deaths registered in the World Health Organization mortality database at December 2009 were extracted for European countries. ${ }^{8}$ The quality of mortality data has been evaluated by the WHO. ${ }^{9}$ England and Wales, Scotland, and Northern Ireland were considered as three separate countries. Cyprus was excluded as data were available for only four years (1999, 2000, 2004, and 2006). Malta was not included because abrupt changes in trends occurred in 1979 and 1995, which seem to coincide with classification changes from ICD-8 (international classification of diseases, eighth revision) to ICD-9 and from ICD-9 to ICD-10. For almost all other countries, data up to 2006 were available, and for some countries, mortality data for 2007 and 2008 were also available.

In addition to the WHO data, we obtained information on mortality statistics for several countries, which allowed enhancement of quality. For Switzerland, a change in coding practice in 1994 resulted in an overestimation of cancer mortality before 1994, and, as 
recommended, we therefore applied a correction factor of 0.85 on rates before $1995 .{ }^{10}$ For Belgium, country-specific mortality was recorded until 1997, but thereafter mortality data were available for Flanders (60\% of the population) and for Brussels $(10 \%$ of the population), whereas for Wallonia (30\% of the population), mortality data were available only for 2004 . For Wallonia, we estimated mortality from 1998 to 2003 and for 2005 by using a linear interpolation calculated with data from 1989 to 2004 available for Flanders and
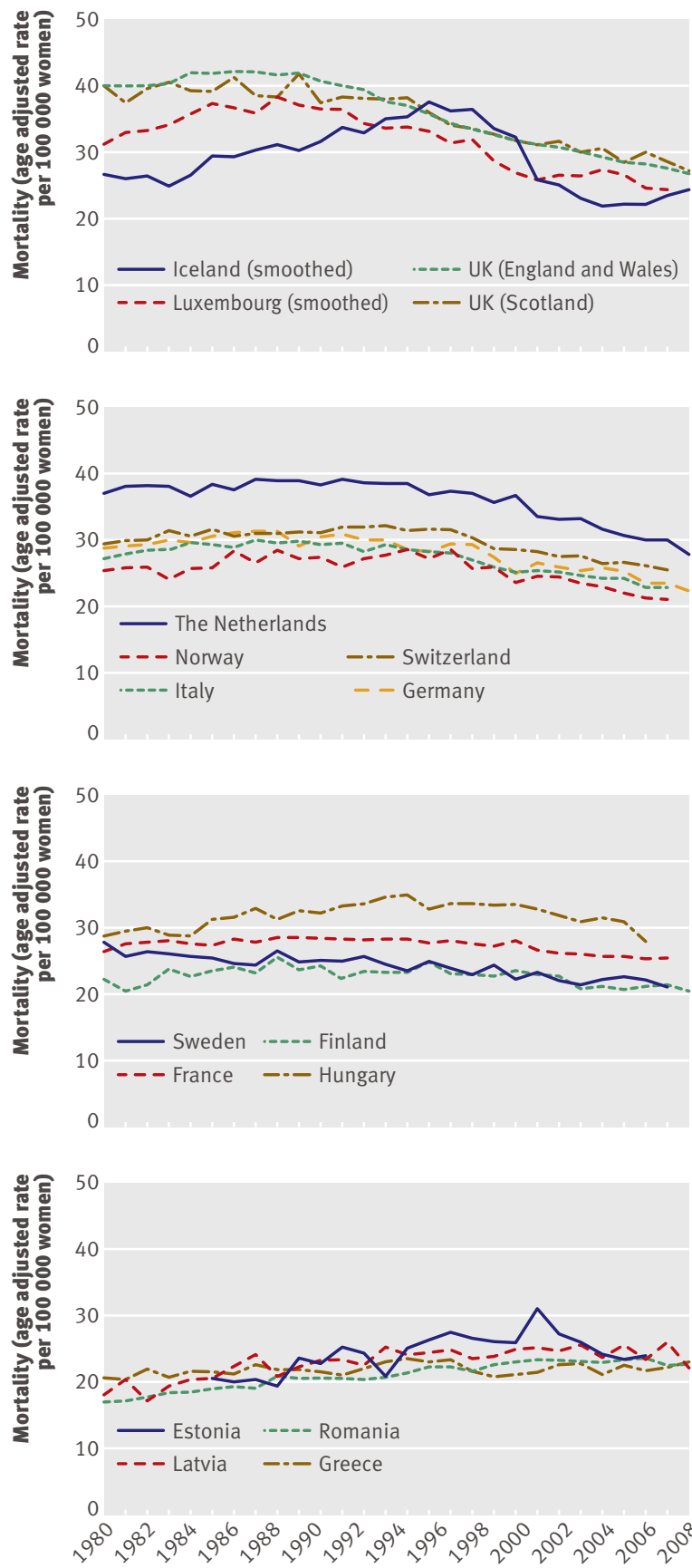

the Brussels region. We subsequently estimated a national mortality for Belgium using regional rates weighted by population size.

In Italy for 2004 and 2005, and in Poland 1997 and 1998, we replaced missing data by interpolations between previous and subsequent calendar years. Mortality values were adjusted by the age distribution of the European standard population ${ }^{11}$ to obtain European standardised rates. Because of small populations, rates in Luxembourg (450 000 inhabitants) and
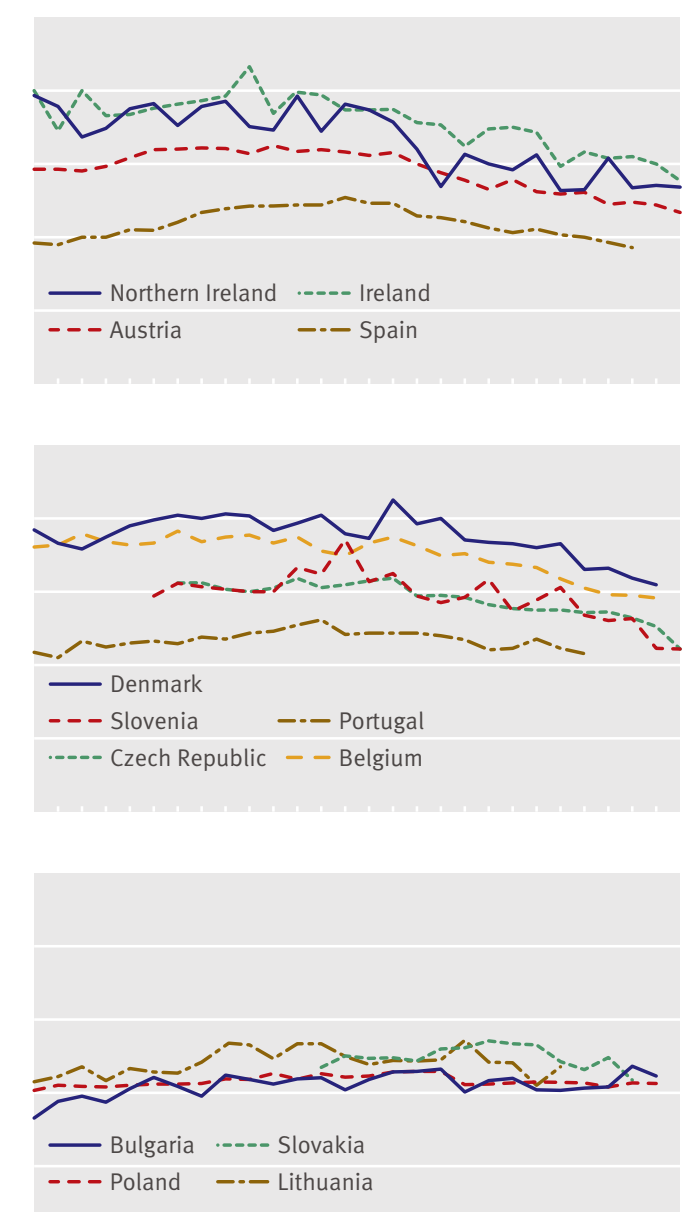

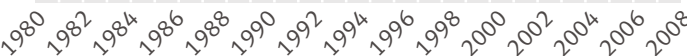

Year

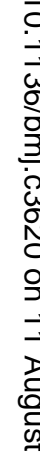

Fig 1 | Temporal trends in breast cancer mortality in European countries. Countries have been grouped in graphs according to percentage change in mortality from 1989 to 2006. (Five-year smoothing was applied on Iceland and Luxembourg rates to avoid unstable trends because of small population size) 
Table 1|Changes in breast cancer mortality between 1989 and 2006 in European countries ranked according to overall decline in mortality

\begin{tabular}{|c|c|c|c|c|c|c|c|c|c|c|c|c|c|}
\hline \multirow[b]{3}{*}{ Country } & \multirow{2}{*}{\multicolumn{2}{|c|}{ Mean mortality* }} & \multicolumn{4}{|c|}{ Mortality change for all ages (\%) } & \multicolumn{6}{|c|}{ Mortality change $1989-2006$ by age group (\%) } & \multirow{3}{*}{$\begin{array}{l}\text { Quality } \\
\text { of data } \\
\text { on cause } \\
\text { of death§} \S\end{array}$} \\
\hline & & & \multicolumn{2}{|c|}{ For $1989-2006$} & \multirow{2}{*}{$\begin{array}{l}\text { Year for } \\
\text { start of } \\
\text { decline‡ }\end{array}$} & \multirow{2}{*}{$\begin{array}{c}\text { Annual } \\
\text { change } \\
1999- \\
2006\end{array}$} & \multicolumn{3}{|c|}{ Annual change } & \multicolumn{3}{|c|}{ Overall change } & \\
\hline & 1987-9 & $2004-6 \dagger$ & Annual & Overall & & & $<50$ & $50-69$ & $\geq 70$ & $\ll 50$ & $50-69$ & $\geq 70$ & \\
\hline Iceland & 33.1 & 23.5 & -3.4 & -44.5 & 1995 & 1.1 & -8.1 & -2.5 & -3.1 & -76.3 & -35.0 & -41.5 & High \\
\hline England and Wales & 41.9 & 28.1 & -2.5 & -34.9 & 1989 & -2.0 & -3.2 & -3.0 & -1.5 & -42.1 & -40.1 & -22.6 & High \\
\hline Luxembourg & 36.3 & 22.9 & -2.4 & -34.1 & 1988 & -2.8 & -5.3 & -2.5 & -1.3 & -60.0 & -34.9 & -19.9 & Medium \\
\hline Scotland & 39.3 & 29.0 & -2.1 & -29.9 & 1990 & -1.4 & -2.9 & -2.7 & -0.7 & -39.1 & -37.2 & -11.9 & High \\
\hline Northern Ireland & 37.0 & 28.1 & -2.0 & -29.2 & 1991 & -1.2 & -3.8 & -2.6 & 0.0 & -48.2 & -36.2 & -0.7 & High \\
\hline Austria & 31.8 & 24.5 & -1.8 & -26.8 & 1990 & -1.6 & -4.0 & -1.7 & -1.1 & -50.3 & -25.3 & -16.9 & Medium \\
\hline Spain & 23.7 & 18.9 & -1.8 & -26.8 & 1992 & -2.2 & -3.4 & -2.1 & -0.3 & -44.7 & -30.3 & -4.6 & Medium \\
\hline Ireland & 40.3 & 30.5 & -1.8 & -26.4 & 1991 & -2.3 & -3.2 & -1.9 & -1.0 & -42.7 & -27.2 & -15.7 & High \\
\hline Netherlands & 39.0 & 30.1 & -1.7 & -25.1 & 1993 & -2.7 & -1.7 & -1.9 & -1.4 & -25.3 & -27.8 & -20.9 & Medium \\
\hline Norway & 27.4 & 21.5 & -1.6 & -24.3 & 1995 & -2.2 & -2.5 & -1.5 & -1.4 & -35.2 & -22.6 & -20.8 & Medium \\
\hline Italy & 29.7 & 23.2 & -1.5 & -22.8 & 1991 & -1.6 & -2.7 & -1.7 & -0.7 & -36.7 & -24.9 & -11.0 & Medium \\
\hline Switzerland $\boldsymbol{T}$ & 30.5 & 24.0 & -1.5 & -22.7 & 1985 & -1.1 & -2.2 & -1.2 & -1.7 & -30.9 & -18.5 & -24.7 & Medium \\
\hline Germany & 31.3 & 26.2 & -1.4 & -21.3 & 1999 & -1.5 & -3.5 & -1.3 & -0.5 & -45.5 & -20.2 & -8.9 & Medium \\
\hline Denmark & 40.5 & 32.0 & -1.4 & -20.8 & 1995 & -2.6 & -3.8 & -1.7 & 0.1 & -48.5 & -25.7 & 1.3 & Medium \\
\hline Belgium & 37.5 & 29.7 & -1.3 & -20.3 & 1986 & -2.4 & -2.7 & -1.5 & -0.4 & -36.7 & -22.0 & -7.2 & Medium \\
\hline Portugal & 23.9 & NA & -1.1 & -17.8 & 1992 & -0.9 & -2.7 & -1.4 & 0.4 & -36.9 & -21.5 & 6.5 & Low \\
\hline Czech Republic & 30.6 & 26.4 & -1.1 & -17.8 & 1994 & -1.2 & -3.7 & -1.7 & 0.5 & -47.2 & -25.5 & 8.6 & Medium \\
\hline Slovenia & 30.7 & 26.3 & -1.0 & -16.1 & 1993 & -2.1 & -4.1 & -1.1 & 0.5 & -51.3 & -17.3 & 9.1 & High \\
\hline Sweden & 25.6 & 22.0 & -1.0 & -16.0 & 1972 & -0.6 & -2.6 & -1.0 & -0.3 & -35.7 & -15.9 & -4.3 & Medium \\
\hline Finland & 24.5 & 21.4 & -0.7 & -11.7 & 1990 & -1.5 & -2.3 & -0.7 & 0.0 & -32.6 & -10.8 & 0.1 & High \\
\hline Hungary & 32.4 & 29.0 & -0.7 & -11.4 & 1994 & -3.1 & -2.4 & -0.5 & -0.1 & -34.4 & -8.3 & -2.4 & High \\
\hline France & 28.5 & 25.6 & -0.7 & -10.7 & 1994 & -1.4 & -0.9 & -0.9 & -0.1 & -14.3 & -14.9 & -1.6 & Medium \\
\hline Poland & 21.5 & 21.1 & -0.4 & -5.9 & None & -0.1 & -2.5 & -0.3 & 0.8 & -34.5 & -4.3 & 14.6 & Low \\
\hline Slovakia & 23.6 & 23.4 & -0.1 & -1.5 & 2000 & -3.2 & -2.1 & -0.1 & 1.1 & -30.7 & -1.9 & 20.5 & High \\
\hline Bulgaria & 21.3 & 22.2 & 0.0 & -0.8 & 1988 & 0.8 & -1.7 & 0.1 & 0.9 & -25.5 & 2.3 & 16.2 & Medium \\
\hline Lithuania & 22.9 & 23.2 & 0.0 & -0.7 & None & -1.1 & -2.7 & -0.3 & 2.7 & -37.0 & -4.3 & 58.1 & High \\
\hline Greece & 21.9 & 22.2 & 0.1 & 1.4 & NR & 0.2 & -3.0 & -1.1 & 3.5 & -40.6 & -17.8 & 80.2 & Low \\
\hline Estonia & 20.9 & 23.6 & 0.5 & 9.6 & NR & -3.1 & -2.0 & 0.6 & 2.2 & -29.0 & 11.4 & 44.9 & High \\
\hline Latvia & 22.5 & 25.1 & 0.6 & 11.4 & NR & 0.0 & -0.9 & 0.6 & 2.2 & -13.7 & 10.2 & 43.6 & High \\
\hline Romania & 19.9 & 23.1 & 0.9 & 16.6 & NR & 0.0 & -1.1 & 0.8 & 2.8 & -17.6 & 14.3 & 59.3 & High \\
\hline \multicolumn{14}{|l|}{ Summary statistics } \\
\hline Median & 30.1 & 24.0 & -1.2 & -19.0 & NR & -1.5 & -2.7 & -1.4 & -0.1 & -36.8 & -20.8 & -2.0 & NR \\
\hline Minimum & 19.9 & 18.9 & -3.4 & -44.5 & NR & -3.2 & -8.1 & -3.0 & -3.1 & -76.3 & -40.1 & -41.5 & NR \\
\hline Maximum & 41.9 & 32.0 & 0.9 & 16.6 & NR & 1.1 & -0.9 & 0.8 & 3.5 & -13.7 & 14.3 & 80.2 & NR \\
\hline
\end{tabular}

$\mathrm{NA}=$ not available; $\mathrm{NR}=$ not relevant.

*Age adjusted (European standard) rate per 100000 women.

tData for 2004-5 for Spain, Hungary, Slovakia, and Estonia; data for 2004 for Belgium; data for 2006 for Italy; 2003 was the last year of data for Portugal.

$\ddagger$ By joinpoint analysis, year of statistical significance at P<0.05 (two sided).

$\S$ Quality of information on cause of death according to WHO (from Mathers et $\mathrm{l}^{9}$ ).

ๆMortality values before 1996 were decreased by $15 \%$ (as recommended by Lutz et $\mathrm{al}^{10}$ ).

Iceland (320000 inhabitants) were unstable, with abrupt changes from one year to another. We therefore smoothed rates of these two countries using a five-year moving average.

We were most interested in recent trends in mortality because efficient treatments and breast screening spread in many European countries in the 1990s. After logarithmic transformation of rates, we fitted a linear regression from 1989 until 2006 and from 1999 until 2006. Because of missing data for the later years, regression was fitted from 1992 until 2005 for Slovakia, and between 1989 and 2004 for Portugal. From the slope of each country, we derived the annual percentage change in breast cancer mortality for the periods
1989-2006 and 1999-2006, and the overall percentage change from 1989 to 2006 . We performed these analyses for women of all ages, and for women aged $<50$, 50-69, and $\geq 70$.

A systematic analysis of temporal trends in women of all ages was done by joinpoint regression using the Joinpoint Regression Program version 3.4.2 from the Surveillance Research Program of the US National Cancer Institute. ${ }^{12}$ Joinpoint regression identifies periods when distinct linear or log-linear trends are separated by inflexion points ("join points"). We performed joinpoint regression on data from 1960 onwards (or from the earliest available data after 1960) until the last year of available data. 


\section{RESULTS}

\section{Overall changes in breast cancer mortality}

Figure 1 and the table show the changes in breast cancer mortality in 30 European countries from 1989 to 2006. Over the 18 years, mortality declined by a median of $19 \%$, with individual values ranging from a $45 \%$ reduction in Iceland to a 17\% increase in Romania. The decrease was $>30 \%$ in three countries, 20\%-30\% in 12 countries, and in four countries breast cancer mortality continued to increase.

In the 15 countries where mortality declined overall by $\geq 20 \%$ the decrease started before 1996, except in Germany, where the decline started in 1999. In Sweden there has been a slow but steady decrease in breast cancer mortality since 1972, without a further joinpoint.

When the 18 year mortality changes were plotted against the mean breast cancer mortality in 1987-9 (fig 2), countries with high mortality in 1987-9 tended to have greater reductions in mortality. However, the decrease varied substantially. In countries with mortality $>35$ per 100000 in 1987-9, decreases ranged from $20 \%$ in Belgium to $35 \%$ in England and Wales. In several countries with mortality in the range of 23-40 per 100000 in 1987-9, there were also substantial reductions in breast cancer mortality-such as $27 \%$ in Spain, 24\% in Norway, and 23\% in Italy - while reductions in Finland, France, and Sweden were 12\%, 11\%, and $16 \%$, respectively.

In countries with the highest mortality reduction, the decline tended to slow during the last eight years of observation. In most other countries, declines after 1998 tended to be more pronounced than for the whole period 1989-2006. In some countries with moderate mortality reductions, however, declines between 1999 and 2006 were less pronounced or even absent, notably in Sweden and Poland. In several central European countries, rates have stabilised or have started to decline after 1998.

With time, breast cancer mortality values for different countries seemed to converge. In 1987-9 mortality (per 100000 ) ranged from 19.9 in Romania to 41.9 in England and Wales (difference 22.0), whereas in 20046 mortality ranged from 18.9 in Spain to 32.0 in Denmark (difference 13.1).

\section{Changes in breast cancer mortality by age group}

Reductions in mortality were most marked in women aged $<50$ years (median change $-37 \%$ (range $-76 \%$ to $-14 \%$ ), and reductions of $14 \%$ or more were observed in all 30 countries. Reductions in women aged 50-69 were similar to those observed in women of all ages $(-21 \%(-40 \%$ to $14 \%)$. The median change in women aged $\geq 70$ was $-2 \%(-42 \%$ to $80 \%)$, with breast cancer mortality continuing to increase in 17 countries. In countries where a mortality reduction could be observed among older women, these reductions were never as robust as for women $<70$ years old. These age related differences were particularly noticeable in central and eastern European countries.

\section{DISCUSSION}

This study shows substantial differences between European countries in temporal trends of breast cancer mortality, as well as differences in mortality changes with age. The sustained decline observed in many countries after 1999 seems to indicate that breast cancer mortality will continue to decrease beyond 2006 . The convergence of annual mortality rates among countries over time was noticed in $2000^{2}$ and could be due to diminishing marginal returns because the countries with the highest mortality in the late 1980s simply had more opportunity for relative change over time.

\section{Strengths and limitations of study}

The cause of death reported in national statistics may be of limited reliability, and WHO quality indices displayed in the table indicate that, for all causes of death combined, data from Greece, Poland, and Portugal should be considered with caution. However, deaths caused by breast cancer may be more reliable than for several other cancers. ${ }^{1314}$ It is possible that some changes in breast cancer mortality trends could be due to changes in coding practices. This problem was encountered in Switzerland, for example. ${ }^{10} \mathrm{We}$ are not aware of similar problems in other European countries, and therefore, we are confident that our results do reflect real trends.

\section{Comparison with other studies}

Our results contrast with those from the EUROCARE study on survival of breast cancer. ${ }^{15}$ The cancer policy of the UK National Health Service has been under scrutiny after the publication of the EUROCARE reports. ${ }^{1617}$ These reports showed that five year relative survival of cancer patients in the UK (mainly patients with breast and prostate cancer and cutaneous

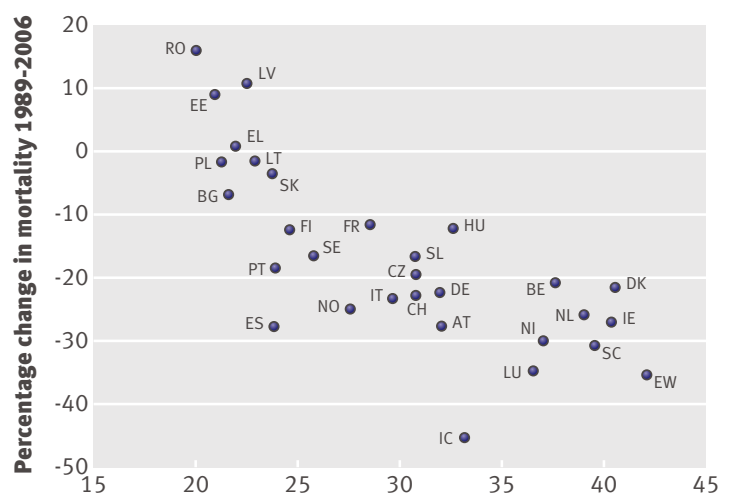

Mean mortality in 1987-9 (age adjusted rate per 100000 women) Key:

$\mathrm{AT}=$ Austria; $\mathrm{BE}=$ Belgium; $\mathrm{BG}=\mathrm{Bulgaria} ; \mathrm{CH}=$ Switzerland; $\mathrm{CZ}=\mathrm{Czech}$ Republic; $\mathrm{DE}=$ Germany; $\mathrm{DK}=$ Denmark; $\mathrm{EL}=\mathrm{Greece} ; \mathrm{EE}=\mathrm{Es}$ stonia; $\mathrm{ES}=\mathrm{Spain}$

$\mathrm{DE}=\mathrm{Germany} ; \mathrm{DK}=\mathrm{Denmark} ; \mathrm{EL}=\mathrm{Greece} ; \mathrm{EE}=\mathrm{ESt}$ tonia; $\mathrm{ES}=\mathrm{Spain} ;$
$\mathrm{EW}=$ England and Wales; $\mathrm{Fl}=$ Finland; $\mathrm{FR}=$ France; $\mathrm{HU}=$ Hungary; $\mathrm{IC}=\mathrm{Iceland} ;$ $\mathrm{IE}=$ Republic of Ireland; IT=Italy; LT=Lithuania; LU=Luxemburg; LV=Latvia; $\mathrm{NI}=$ Northern Ireland; $\mathrm{NL}=$ Netherlands; $\mathrm{NO}=$ Norway; $\mathrm{PL}=$ Poland; $\mathrm{PT}=$ Portugal; $\mathrm{RO}=$ Romania; $\mathrm{SC}=$ Scotland; $\mathrm{SE}=$ Sweden; $\mathrm{Sl}=$ Slovenia; $\mathrm{SK}=$ Slovakia

Fig 2 | Percentage changes in breast cancer mortality in European countries during 1989-2006 according to the mean breast cancer mortality in 1987-9 
melanoma) was lower than in neighbouring countries despite significant investment in services. ${ }^{15}$ In addition, a report on access to cancer drugs in Europe concluded that the lower survival of breast cancer patients in the UK could be due to delay in licensing new drugs. ${ }^{6} 18$

While mortality and survival data have their limitations, mortality statistics are indicative of long term trends, whereas the survival statistics can be severely biased by changing trends in the incidence of small cancers with a good prognosis, as will typically happen when screening is widespread. Between 1990 and 2002 , breast cancer incidence increased by $20 \%-48 \%$ in most countries where five year relative survival was estimated to be $\geq 80 \% .{ }^{151920}$ In the UK and Scotland, the increases in incidence were $16 \%$ and $13 \%$, respectively. ${ }^{1920}$

Hence, survival differences mainly stem from the higher incidence of breast cancer in countries with higher survival. ${ }^{21}$ The substantial increase in breast cancer incidence observed after 1990 in many European countries was partly due to advances in diagnosis (lead time) and to increasing detection of nonprogressive or slowly progressing tumours with no or weak life threatening potential (length time). ${ }^{22}$ Lead time and length time were to a large extent introduced by screening activities that led to increasing incidence of early stage breast cancers. ${ }^{23-25}$ Lead time and length time biases will spuriously modify survival even in the absence of a change in mortality. ${ }^{21}$ Differences in survival may therefore reflect differences in screening intensity, and not reductions in mortality. Therefore, the UK NHS cancer policy was successful in reducing breast cancer mortality, and the rather bleak picture of UK cancer policy portrayed using breast cancer survival is likely to be erroneous.

\section{Interpretation of results}

At least four factors may explain the considerable variations in breast cancer mortality between European countries. Firstly, mortality levels at the end of the 1980s could have influenced some of the relative differences in mortality reductions, but they cannot account for a large part of variations between countries.

Secondly, the incidence of breast cancer has increased in most European countries over the past 20 years, ${ }^{1920}$ resulting in doctors mentioning breast cancer more frequently as the cause of death. Also, in populations with a substantial increase in cancer incidence more advanced cancers may have occurred that have probably attenuated the mortality decline. The sharp increases in incidence observed after 1985 in many white populations were mainly due to the combined effects of changes in reproductive factors, overweight and obesity, screening mammography, and hormone replacement therapy. ${ }^{26-28}$ Most of these factors do not have the same impact on risk of breast cancer as on death from breast cancer. ${ }^{29}$ For instance, breast screening is deemed to reduce breast cancer mortality, but it also increases the incidence of cancers with low aggressive potential. ${ }^{25}{ }^{30-33}$ Also, a closer look at both incidence and mortality data in a large number of countries with national cancer registries shows that there is only a weak association between changes in incidence and changes in mortality. ${ }^{1920}$

Thirdly, breast screening is expected to decrease the median size of breast cancers, resulting in steadily earlier stage at diagnosis and lower mortality. ${ }^{345}$ It is, however, unclear whether the reductions in median breast cancer size observed since the 1980s result from a general decrease in size, with concomitant decrease in the incidence of advanced cancer, or rather from the increasing incidence of small, early cancers that would give the overall impression of downsizing. ${ }^{2336-38}$

Fourthly, adoption of efficient treatment regimens and reorganisation of health services that encourages a multidisciplinary approach and high volume specialised breast cancer clinics may have benefited women irrespective of whether their cancer was detected by screening. Few European countries, however, have published time trends in hormone therapy and chemotherapy delivered to patients or published information on breast cancer management. ${ }^{39-45}$ Recently published data comparing spending on new cancer drugs and their uptake ${ }^{6}$ do not assess treatment efforts specific to breast cancer, including changes in nondrug treatments (such as changes in surgical and radiotherapy management). Mortality reductions started after the release of reports on efficacy of chemotherapy and hormone therapy. ${ }^{46}$ Similar reductions in mortality were observed in women aged $<50$ years in countries where screening of young women has been rare (Denmark, UK, Netherlands, Norway, Finland) and where it is common (Sweden, France, Germany). ${ }^{3-48}$ Hence, the particularly larger reduction in mortality in women $<50$ years old may reflect better targeting of effective treatments and response to treatments.

The smaller decrease or continued increase in breast cancer mortality seen in women $\geq 70$ years old may be due to lower awareness, more frequent diagnosis of cancer at an advanced stage, and less intensive treatment of cancer. ${ }^{4049-52}$

\section{Conclusions and policy implications}

The largest decreases in mortality were expected in countries that combined high mortality in the late 1980s, high participation in nationwide screening starting early in the 1990s, rapid adoption and easy access to new treatments, and restructuring of medical services towards high volume specialised breast clinics and multidisciplinary management. Data on all these factors have been published for the UK, showing high screening coverage of women aged 50-64 after 1995, ${ }^{53}$ a rapid and general use of reasonably priced tamoxifen and adjuvant chemotherapy by UK doctors, ${ }^{5455}$ and the reorganisation of breast cancer services on the basis of the Calman-Hine and Campbell evaluation reports. $^{55-58}$

At the other end of the spectrum, in central European countries the small decreases or the continued increase in breast cancer mortality were correlated with low, usually non-organised, screening activities, ${ }^{3}$ 


\section{WHAT IS ALREADY KNOWN ON THIS TOPIC}

Since 1985-95, breast cancer mortality has been falling in many European countries, with the decreases attributed to the combined effects of breast screening and better treatments

Cancer plans in the UK have been criticised because of the lower survival of cancer patients compared with other European countries.

\section{WHAT THIS STUDY ADDS}

The major decreases in breast cancer mortality observed in the UK were more marked than in most European countries and were only partly linked to high mortality at the end of the 1980s.

Mortality data are a more realistic measure of successful cancer control than cancer survival, as the latter is influenced by changes in cancer incidence

Some countries, such as France and Sweden, have mobilised large resources on screening and acquisition of cancer drugs but have shown little change in breast cancer mortality

Breast cancer mortality is still increasing in several central European countries, representing avoidable cancer deaths
Funding: Institutions in which authors work had no influence in the conduct of the research or in the writing of the paper. The work of the Northern Ireland Cancer Registry is funded by the Public Health Agency for Northern Ireland

Competing interests: All authors have completed the Unified Competing Interest form and declare no financial or non-financial interests that may be relevant to the submitted work.

Ethical approval: Not required.

Data sharing: The WHO mortality database is available at www.WHO.int.

1 Hermon C, Beral V. Breast cancer mortality rates are levelling off or beginning to decline in many western countries: analysis of timetrends, age-cohort and age-period models of breast cancer mortality in 20 countries. Br J Cancer 1996; 73:955-60.

2 Levi F, Bosetti C, Lucchini F, Negri E, La Vecchia C. Monitoring the decrease in breast cancer mortality in Europe. Eur J Cancer Prev 2005;14:497-502.

3 European Commission. Cancer screening in the European Union. Report on the implementation of the council recommendation on cancer screening. First report. 2008. http://ec.europa.eu/health/ ph_determinants/genetics/documents/cancer_screening.pdf.

4 Coebergh JW, Albreth T. Should the EU also wage war against cancer? And if so, how? Eur / Cancer 2008;44:1341-4.

5 Micheli A, Coebergh JW, Mugno E, Massimiliani E, Sant M, Oberaigner W, et al. European health systems and cancer care. Ann Oncol 2003;14 (suppl 5):v41-60.

low numbers of mammography machines, ${ }^{7}$ slow uptake of anticancer drugs, ${ }^{6}$ and health expenditures below the European average. ${ }^{59}$ These epidemiological patterns may also be linked to rapid changes in breast cancer risk factors that took place in these countries after the collapse of the communist regimes in the 1990 s, such as decreasing fertility and increasing age at first birth. ${ }^{60}$

In some countries, more impressive changes in mortality were expected in view of the efforts and resources devoted to combating breast cancer. For instance, the decline in breast cancer mortality in France has been quite modest despite this country having one of the highest number of mammography units per million women, ${ }^{7}$ having the highest spending on cancer drugs per person in Europe, ${ }^{6}$ being at the forefront in use of new costly treatments, ${ }^{6}$ and devoting much effort to enhancing adherence to guidelines. ${ }^{61}$

In Sweden, the $16 \%$ decrease in mortality between 1989 and 2005 includes reductions obtained with screening, treatments, and system efficiency, and this decrease has remained stable since 1972. Observational studies that included about $60 \%$ of the Swedish female population have shown that screening alone could have reduced the risk of death from breast cancer by $25 \%-30 \%$ in all women $\geq 40$ years old. ${ }^{6263}$ Given the these results and given that breast screening was also implemented in areas that were not part of these observational studies, one would have expected a better match between mortality statistics and the results from these studies.

Clearly, improving the collection of relevant screening and management data is warranted for understanding the strong variations in breast cancer mortality in Europe. Efforts should be made to reduce the avoidable breast cancer mortality in central European countries.

Contributors: Study concept and design: PA. Data collection: PA, MH, MB. Data analysis: PA, CH, MB. Data interpretation: all authors. Writing of manuscript: PA, CLV, AG, LV. Editing of final version and approval for submission: all authors. PA is the guarantor of the study.
6 Jonsson B, Wilking N. A global comparison regarding patient access to cancer drugs. Ann Oncol 2007;18(suppl 3):1-74S.

7 Autier P, Ouakrim DA. Determinants of the number of mammography units in 31 countries with significant mammography screening. Br J Cancer 2008;99:1185-90.

8 World Health Organization. Mortality database. World Health Organization, 2009.

9 Mathers CD, Ma Fat D, Inoue M, Rao C, Lopez A. Counting the dead and what they died from: an assessment of the global status of caus of death data. Bull World Health Org 2005;83:171-7.

10 Lutz JM, Pury P, Fioretta G, Raymond L. The impact of coding process on observed cancer mortality trends in Switzerland. Eur J Cancer Prev 2004; 13:77-81.

11 Waterhouse J, Muir C, Correa P, Powel J, eds. Cancer incidence in five continents. Vol III. IARC scientific publication number 15. World Health Organization, 1976.

12 National Cancer Institute. Joinpoint regression program, version 3.4.2. 2009. www.srab.cancer.gov/joinpoint/.

13 Doll R, Peto R. The causes of cancer. Oxford University Press, 1981.

14 Nystrom L, Larsson LG, Rutqvist LE, Lindgren A, Linqdvist M, Ryden S, et al. Determination of cause of death among breast cancer cases in the Swedish randomized mammography screening trials. A committee. Acta Oncol 1995:34:145-52.

15 Berrino F, De Angelis R, Sant M, Rosso S, Bielska-Lasota M Coebergh JW, et al. Survival for eight major cancers and all cancers combined for European adults diagnosed in 1995-99: results of the EUROCARE-4 study. Lancet Oncol 2007;8:773-83.

16 Does the UK really have an effective cancer plan? Lancet Oncol 2007;8:747.

17 Cancer treatment: not just a question of costs. Lancet 2007;369:1665.

18 Jonsson B. Being NICE is not the problem! Eur J Cancer 2009; 45:1100-2.

19 Héry C, Ferlay J, Boniol M, Autier P. Changes in breast cancer incidence and mortality in middle-aged and elderly women in 28 countries with Caucasian majority populations. Ann Oncol 2008;19:1009-18.

20 Héry C, Ferlay J, Boniol M, Autier P. Quantification of changes in breast cancer incidence and mortality since 1990 in 35 countries with Caucasian-majority populations. Ann Oncol 2008;19:1187-94.

21 Autier $\mathrm{P}$, Boniol M, Héry C, Masuyer E, Ferlay J. Cancer survival statistics should be viewed with caution. Lancet Oncol 2007;8:1050-2.

22 Biesheuvel C, Barratt A, Howard K, Houssami N, Irwig L. Effects of study methods and biases on estimates of invasive breast cance over-detection with mammography screening: a systematic review. Lancet Oncol 2007;8:1129-38.

23 Fracheboud I, Otto SJ, van Dijck JA, Broeders MJ, Verbeek AL, de Koning HJ. Decreased rates of advanced breast cancer due to mammography screening in The Netherlands. Br J Cancer 2004:91:861-7.

24 Jemal A, Ward E, Thun MJ. Recent trends in breast cancer incidence rates by age and tumor characteristics among US women. Breast Cancer Res 2007;9:R28.

25 Chiu C, Morrell S, Page A, Rickard M, Brassil A, Taylor R. Populationbased mammography screening and breast cancer incidence in New South Wales, Australia. Cancer Causes Control 2006;17:153-60. comparison between official statistics and validation by an endpoint 
26 McPherson K, Steel CM, Dixon JM. ABC of breast diseases. Breast cancer epidemiology, risk factors, and genetics. $B M$ J 2000;321:624-8.

27 Veronesi U, Boyle P, Goldhirsch A, Orecchia R, Viale G. Breast cancer. Lancet 2005;365:1727-41.

28 Kumle M. Declining breast cancer incidence and decreased HRT use. Lancet 2008;372:608-10.

29 Barnett GC, Shah M, Redman K, Easton DF, Ponder BA, Pharoah PD. Risk factors for the incidence of breast cancer: do they affect survival from the disease? J Clin Oncol 2008;26:3310-6.

30 Zahl PH, Mæhlen J, Welch HG. The natural history of invasive breast cancers detected by screening mammography. Arch Intern Med 2008;168:2311-6.

31 Jørgensen KJ, Gøtzsche PC. Overdiagnosis in publicly organised mammography screening programmes: systematic review of incidence trends. BMJ 2009;339:b2587.

32 Morrell S, Barratt A, Irwig L, Howard K, Biesheuvel C, Armstrong B. Estimates of overdiagnosis of invasive breast cancer associated with screening mammography. Cancer Causes Control 2010;21:275-82.

33 Burke JP, Power C, Gorey TF, Flanagan F, Kerin MJ, Kell MR. A comparative study of risk factors and prognostic features between symptomatic and screen detected breast cancer. Eur J Surg Oncol 2008;34:149-53.

34 Day NE, Williams DR, Khaw KT. Breast cancer screening programmes: the development of a monitoring and evaluation system. Br J Cancer 1989;59:954-8.

35 Autier P, Héry C, Haukka J, Boniol M, Byrnes G. Advanced breast cancer and breast cancer mortality in randomized controlled trials on mammography screening. J Clin Oncol 2009;27:5919-23.

36 National Evaluation Team for Breast Cancer Screening. National evaluation of breast cancer screening in the Netherlands. 11th evaluation report. NETB, 2005.

37 McCann J, Stockton D, Day N. Breast cancer in East Anglia: the impact of the breast screening programme on stage at diagnosis. J Med Screen 1998;5:42-8.

38 Hofvind S, Sorum R, Thoresen S. Incidence and tumor characteristics of breast cancer diagnosed before and after implementation of a population-based screening program. Acta Oncol 2008;47:225-31.

39 Vervoort MM, Draisma G, Fracheboud J, van de Poll-Franse LV, de Koning HJ. Trends in the usage of adjuvant systemic therapy for breast cancer in the Netherlands and its effect on mortality. $\mathrm{Br}$ Cancer 2004;91:242-7.

40 Sukel MP, van de Poll-Franse LV, Nieuwenhuijzen GA, Vreugdenhil G, Herings RM, Coebergh JW, et al. Substantial increase in the use of adjuvant systemic treatment for early stage breast cancer reflects changes in guidelines in the period 1990-2006 in the southeastern Netherlands. Eur J Cancer 2008;44:1846-54.

41 Women's Health Council. Breast cancer treatments: a review of the bio-medical evidence. 2008. www.whc.ie/documents/ 28_breastCancerFactsheet08.pdf

42 Valentini M, Mari E, Belfiglio M, Nicolucci A. Is adjuvant tamoxifen used optimally in the treatment of breast cancer? Results of an Italian survey. Ann Oncol 1999;10:789-93.

43 Abadie C, Aminot I, Dupuy E, Degré A. Breast cancer II. [An evaluation of the diagnostic and therapeutic decisions in Aquitaine in 1999. Rev Méd Assurance Maladie 2002;33:267-75.

44 Fritsch B, Ostermann M, Thorn, Seiller F, Fischer B, Weill G. [Assessment of management practices for women with early-stage breast cancer.] La Presse Médicale 2007;36:1712-20.

45 Madhavan KS, Murray PA. A UK survey of tamoxifen prescribing and oestrogen receptor status. Breast 2002;11:265-6.
46 Early Breast Cancer Trialists' Collaborative Group. Effects of adjuvant tamoxifen and of cytotoxic therapy on mortality in early breast cancer: an overview of 61 randomised trials among 28,896 women. N Engl J Med 1988;319:1681-92.

47 Klug SJ, Hetzer M, Blettner M. Screening for breast and cervical cancer in a large German city: participation, motivation and knowledge of risk factors. Eur I Public Health 2005;15:70-7.

48 Spyckerelle Y, Kuntz C, Giordanella JP, Ancelle-Park R. Pratiques de la mammographie chez les femmes de 35 à 75 ans: étude descriptive dans la population consultant les centres d'examens de santé. Bulletin du Cancer 2002;89:957-62.

49 August DA, Rea T, Sondak VK. Age-related differences in breast cancer treatment. Ann Surg Oncol 1994;1:45-52.

50 Eaker S, Dickman PW, Bergkvist L, Holmberg L. Differences in management of older women influence breast cancer survival: results from a population-based database in Sweden. PLoS Med 2006;3:e25.

51 Siebel MF, Muss HB. The influence of aging on the early detection, diagnosis, and treatment of breast cancer. Curr Oncol Rep 2005;7:23-30.

52 Bernardi D, Errante D, Galligioni E, Crivellari D, Bianco A, Salvagno L, et al. Treatment of breast cancer in older women. Acta Oncol 2008;47:187-98.

53 NHS Cancer Screening Programmes. NHS breast screening programme. 2006. www.cancerscreening.nhs.uk/breastscreen/ index.html.

54 Bradburn MJ, Altman DG, Smith P, Fentiman IS, Rubens RD. Time trends in breast cancer survival: experience in a single centre, 1975 89. BrJ Cancer 1998;77:1944-9.

55 Pisani P, Forman D. Declining mortality from breast cancer in Yorkshire, 1983-1998: extent and causes. Br J Cancer 2004;90:652-6.

56 Morris E, Haward RA, Forman D. The impact of the Calman-Hine report: analysis of breast and colorectal cancer surgical workloads and the degree of surgical site specialization in the Yorkshire region of the UK, 1990-2000. Eur J Cancer Care 2007;16:150-5.

57 Morris E, Haward RA, Gilthorpe MS, Craigs C, Forman D. The impact of the Calman-Hines report on the processes and outcomes of care fo Yorkshire's breast cancer patients. Ann Oncol 2008;19:284-91.

58 Monaghan P, Murray L, Donnelly M, et al. Breast cancer services-a population-based study of service reorganization. J Public Health 2005;27:171-5.

59 World Health Organization. WHO statistical information system. 2009. www.who.int/whosis/.

60 Sobotka T. Re-emerging diversity: rapid fertility changes in Central and Eastern Europe after the collapse of the communist regimes. Population 2003;58:451-86.

61 Ray-Coquard I, Philip T, de Laroche G, Froger X, Suchaud IP, Voloch A, et al. Persistence of medical change at implementation of clinical guidelines on medical practice: a controlled study in a cancer network. J Clin Oncol 2005;23:4414-23.

62 Swedish Organised Service Screening Evaluation Group. Reduction in breast cancer mortality from organized service screening with mammography: 1. Further confirmation with extended data. Cance Epidemiol Biomarkers Prev 2006;15:45-51.

63 Swedish Organised Service Screening Evaluation Group. Reduction in breast cancer mortality from organized service screening with mammography: 2. Validation with alternative analytic method. Cancer Epidemiol Biomarkers Prev 2006;15:52-6.

Accepted: 21 May 2010 